\title{
Factors affecting hospital response in biological disasters: A qualitative study
}

\author{
Simintaj Sharififar ${ }^{1,2}$, Katayoun Jahangiri*3,1미 , Armin Zareiyan ${ }^{4}$, Amir Khoshvaghti $^{5}$ \\ Received: 6 Mar $2019 \quad$ Published: 16 Mar 2020
}

\begin{abstract}
Background: The fatal pandemics of infectious diseases and the possibility of using microorganisms as biological weapons are both rising worldwide. Hospitals are vital organizations in response to biological disasters and have a crucial role in the treatment of patients. Despite the advances in studies about hospital planning and performance during crises, there are no internationally accepted standards for hospital preparedness and disaster response. Thus, this study was designed to explain the effective factors in hospital performance during biological disasters.

Methods: Qualitative content analysis with conventional approach was used in the present study. The setting was Ministry of Health and related hospitals, and other relevant ministries responsible at the time of biologic events in Islamic Republic of Iran (IR of Iran) in 2018. Participants were experts, experienced individuals providing service in the field of biological disaster planning and response, policymakers in the Ministry of Health, and other related organizations and authorities responsible for the accreditation of hospitals in IR of Iran. Data were collected using 12 semi-structured interviews in Persian language. Analysis was performed according to Graneheim method.

Results: After analyzing 12 interviews, extraction resulted in 76 common codes, 28 subcategories, and 8 categories, which are as follow: detection; treatment and infection control; coordination, Resources; training and exercises; communication and information system; construction; and planning and assessment.

Conclusion: Hospital management in outbreaks of infectious diseases (intentional or unintentional) is complex and requires different actions than during natural disasters. In such disasters, readiness to respond and appropriate action is a multifaceted operation. In IR of Iran, there have been few researches in the field of hospital preparation in biologic events, and the possibility of standardized assessment has be reduced due to lack of key skills in confronting biological events. It is hoped that the aggregated factors in the 8 groups of this study can evaluate hospital performance more coherently.
\end{abstract}

Keywords: Key capabilities, Biological disasters, Assessment, Performance, Preparedness, Hospital

Conflicts of Interest: None declared

Funding: None

*This work has been published under CC BY-NC-SA 1.0 license.

Copyright $\odot$ Iran University of Medical Sciences

Cite this article as: Sharififar S, Jahangiri K, Zareiyan A, Khoshvaghti A. Factors affecting hospital response in biological disasters: A qualitative study. Med J Islam Repub Iran. 2020 (16 Mar);34:21. https://doi.org/10.47176/mjiri.34.21

\section{Introduction}

The deadly pandemics of infectious diseases are rising

Corresponding author: Dr. Katayoun Jahangiri, k.jahangiri@sbmu.ac.ir

1. Department of Health in Disasters and Emergencies, School of Public Health and Safety, Shahid Beheshti University of Medical Sciences, Tehran, Iran

2. School of Nursing, Aja University of Medical Sciences, Tehran, Iran

3. Safety Promotion and Injury Prevention Research Center, Shahid Beheshti University of Medical Sciences, Tehran, Iran

4. Department of Health in Disasters and Emergencies, School of Nursing, Aja University of Medical Sciences, Tehran, Iran

5. Infectious Diseases Research Center, Aerospace and Subaquatic Medicine Faculty, Aja University of Medical Sciences, Tehran, Iran worldwide. In the twentieth century, 3 influenza pandemics

\section{$\uparrow$ What is "already known" in this topic:}

Infectious epidemics and pandemics threat the public health of societies and countries. Intentional use of biologic factors has had an increasing trend, and hospitals' preparedness and response to such factors has an active role in decreasing morbidity, infection transmission, and mortality.

\section{$\rightarrow$ What this article adds:}

In this study, the factors affecting hospitals' performance in biological disasters from viewpoint of key and informant persons and health politicians have been extracted using content analysis in a qualitative study. It is recommended to evaluate hospitals' performance during disasters to increase their level of preparedness and performance. 
have caused death of more than 50 million people in many parts of the world (1). In the last relatively mild H1N1 pandemic in 2009, $150000-580000$ of people were killed who were not necessarily in high-risk groups (2). According to the report of www.warontherocks.com, about 450 CBRN events were reported between 1990 and 2013 (3). Also, the possibility of using microorganisms as biological weapons is a real and increasing probability all around the world $(4,5)$. Increasing the tendency to use biological weapons due to increased terrorist attacks, their relative convenience use, and low cost have led to many health concerns $(6,7)$. Between 2004 and 2017, 1300 - 1500 persons were killed in major terrorist incidents nearly every 6 months (8).

Hospitals are vital organizations during biological disasters and play a crucial role as a place of care and treatment for such patients $(4,9,10)$. Hospitals should have an essential role in biological disasters (11-14). Having specialists and staff with knowledge and skills relevant to biological events can play a significant role in reducing mortality and morbidity in the community, especially in the first few hours, which is called the golden time. The result is hospital preparedness to deal with biological events, which improves the response rate and accelerates the process of rehabilitation (9). Hospital services are differentiated from other institutions by their types of activities, resources, staff, multiple specializations, and equipment used (10). The response of hospitals has a multidimensional function (approach) $(15,16)$. Despite the advances in studies about hospital planning and performance during crises, there are no internationally accepted standards for hospital preparedness and disaster response (17). To date, there has been no valid methodology for assessing the preparedness of hospitals for disasters (18). However, after the onset of disasters, it is necessary that hospitals be prepared to deal with the new circumstance and surge capacity $(19,20)$.

Disaster preparedness is recognized as one of the top priorities in the medical field (21). This process varies in CBRN events (9). According to the recommendation in the process of planning, preparing, and responding to disasters in the health system, it is necessary to use the "all-hazards" approach (22). However, in practice, this approach does not seem to be suitable for man-made and technologic disasters such as biological, chemical, or nuclear events. Nevertheless, evaluating hospitals' performance during crises, especially the one caused by biological disasters (eg, the epidemic of diseases whether natural or intentional), is a topic that has been dealt with inadequately. Since in the face of disasters and biological threats different conditions prevail in hospitals, thus, evaluating the performance of hospitals in such situations requires a different mechanism. In addition, no comprehensive plan has been developed to manage biological events in Iran. Therefore, the present study was conducted to identify factors affecting hospital response in biological disasters.

\author{
Methods \\ Design \\ Qualitative content analysis with conventional approach
}

was used in this study. The qualitative content analysis approach was used for subjective interpretations of text data by systematic classification process, coding, identifying categories, or patterns. Using this approach, the researcher avoids classification with background thoughts and allows categories formation during the research process (23-25). Hospitals under the supervision of Ministry of Health and other relevant ministries responding to biologic events in IR of Iran at 2018 (including the Defense Ministry) were included in this study.

\section{Study participants and data collection}

Participants in this project were experts, experienced individuals providing services in the field of biological disaster planning and response, policymakers in Ministry of Health, and other related organizations and authorities responsible for the accreditation of hospitals in IR of Iran. Data were collected through conducting semi-structured interviews in Persian language with the aim of explaining factors affecting the performance of hospitals in response to biologic threats.

Initially, the researcher met each participant and presented the research goals and obtained their consent for participation. Three participants did not respond to the request and 4 introduced another person as an expert in the field of research. Two participants stated that these questions were not in their field of expertise. A total of 12 interviews were done. The age range of the participants was 38-62 years.

The purpose of the interviews was to explain and explore the factors influencing hospital performance in response to biologic threats. Interviews began with simple and general topics and went on to specific questions. Some questions were changed during the research (after completing the third interview and analyzing the data). Types of questions were as follow: open questions, based on the default, and case-by-case. Some of the interview questions were as follow:

1. What are the effective factors in assessing hospital performance at the time of biological events?

2. What are the management problems that you have experienced or may experience during a biological threat?

3. What are the strengths and weaknesses in assessing a hospital's performance of biological threats?

4. What is the difference between assessing the performance of hospitals in natural disasters and in biological distasters?

During the interview, the researcher observed and noted the participants' interactions with the environment and their reactions, which were considered in the data analysis. The number of participants was determined based on the saturation of the obtained codes, so that the new codes were not extracted by new interviews. Sampling was done using purposeful and snowball method. The interview took about 50 110 minutes.

\section{Data analysis}

At first, interviews were conducted with the participants, their voice was recorded, and transcription was done a short while after each interview. Data analysis began after the 
first transcription. To analyze the data, the researcher studied the data deeply, reread, and considered the text of the interview, gained understanding of the data, and finally completed the analysis (23).

After each interview, transcription was done and field observations notes were reviewed several times. The final text was approved by each participant (member check). After the third interview, the questions were redesigned. In this study, interviews and field notes were the analysis units. The texts were divided into content areas and meaning units. Meaning units were summarized and codes were extracted. Multiple codes were compared with each other in terms of differences and similarities; then, categories and subcategories were formed. The extracted categories were discussed by 2 scholars; finally, the basic and essential meanings of the categories were edited. Data collection continued until saturation was reached for each concept.

\section{Rigor}

In a qualitative research, rigor shows concepts of credibility, dependability, transferability, and different dimensions of trustworthiness (23). To achieve maximum credibility, the research team used a range of expert participants. Participants were among authorities of Health in IR of Iran, officials and staff of biologic laboratories, and some experienced officials from civil and military organizations with a history of managing infectious disasters.

After each interview, transcription of texts was performed in a short interval. Interviews were listened repeatedly, and the researcher extracted the meaning units. Then, based on the condensation and abstracting of meaning units, the codes were extracted. Similar codes were placed in subcategories, and the categories were formed according to the similarities and differences between subcategories.

In the case of extraction of codes, subcategories, and categories, expert opinions were taken from the research team, and agreement was reached among researchers, experts, and participants about the differences. This study has been conducted in the context of IR of Iran. According to researchers' view, the results can be generalized to other countries, but the choice has been left to readers (23).

\section{Ethics}

This study was approved by the National Committee of Ethics of IR of Iran (code number: IR.SBMU.RETECH. REC.1396.205). All participants were aware of the research objectives. Informed consent was taken from all participants and their participation was kept confidential. All interviews were recorded with participants' permission and were fully transcribed within a short period after the interview. Analysis was done simultaneously.

\section{Results}

The demographic characteristics of the participants are presented in Table 1.

Participants answered all the questions during the interview. After analyzing 12 interviews, 76 codes were extracted. Codes were categorized in 28 subcategories after analysis. According to the similarities and differences in nature of subcategories, 8 categories were extracted: detection; treatment and infection control; coordination; resources; training and exercises; communication and information system; construction; and planning and assessment.

The graphic diagram of the extracted categories and subcategories is shown in Figure 1.

Table 2 contains extracted categories, subcategories, and common codes by content analysis.

\section{Detection}

After analyzing the data, it was found that all participants considered the ability to detect biologic outbreaks or emergencies in hospitals as an effective factor in hospital performance. The first step in controlling a biologic emergency is to detect the event in a hospital. The subcategories of determining the type of event (intentional or unintentional) and early detection were extracted.

1-1: According to the participants, the delay in diagnosis with subsequent possibility of developing outbreaks of communicable diseases could be a major factor in hospital performance.

One of the participants (P03) mentioned, "Rapid detection is an important factor in proper performance in biological disasters. In the Crimean-Congo fever epidemic, if diagnosis was not established on time, the disease would have spread further".

1-2: Determining the deliberate or unintentional cause of a biological event can affect hospital performance. In intentional events, the biologic agent may still exist in the environment which will cause the disaster to be continued.

Another participant (P02) said, "It is difficult to diagnose the cause of deliberate biological events because there is less experience about it. Treatment has been discussed on

Table 1. Demographic characteristics of the participants

\begin{tabular}{llcc}
\hline Demographic variable & & Number & Frequency (\%) \\
\hline Sex & Female & 3 & 25 \\
\multirow{5}{*}{ Age } & Male & 9 & 75 \\
& $30-40$ & 4 & 33.5 \\
\multirow{5}{*}{ Education } & $40-50$ & 5 & 41.5 \\
\multirow{2}{*}{ Organization } & $\geq 50$ & 3 & 25 \\
& PhD & 6 & 50 \\
& Medical specialist & 6 & 50 \\
& Ministry of Health & 2 & 17 \\
& Ministry of Defense & 1 & 8 \\
& Hospital & 6 & 50 \\
& Research center & 3 & 25 \\
\hline
\end{tabular}



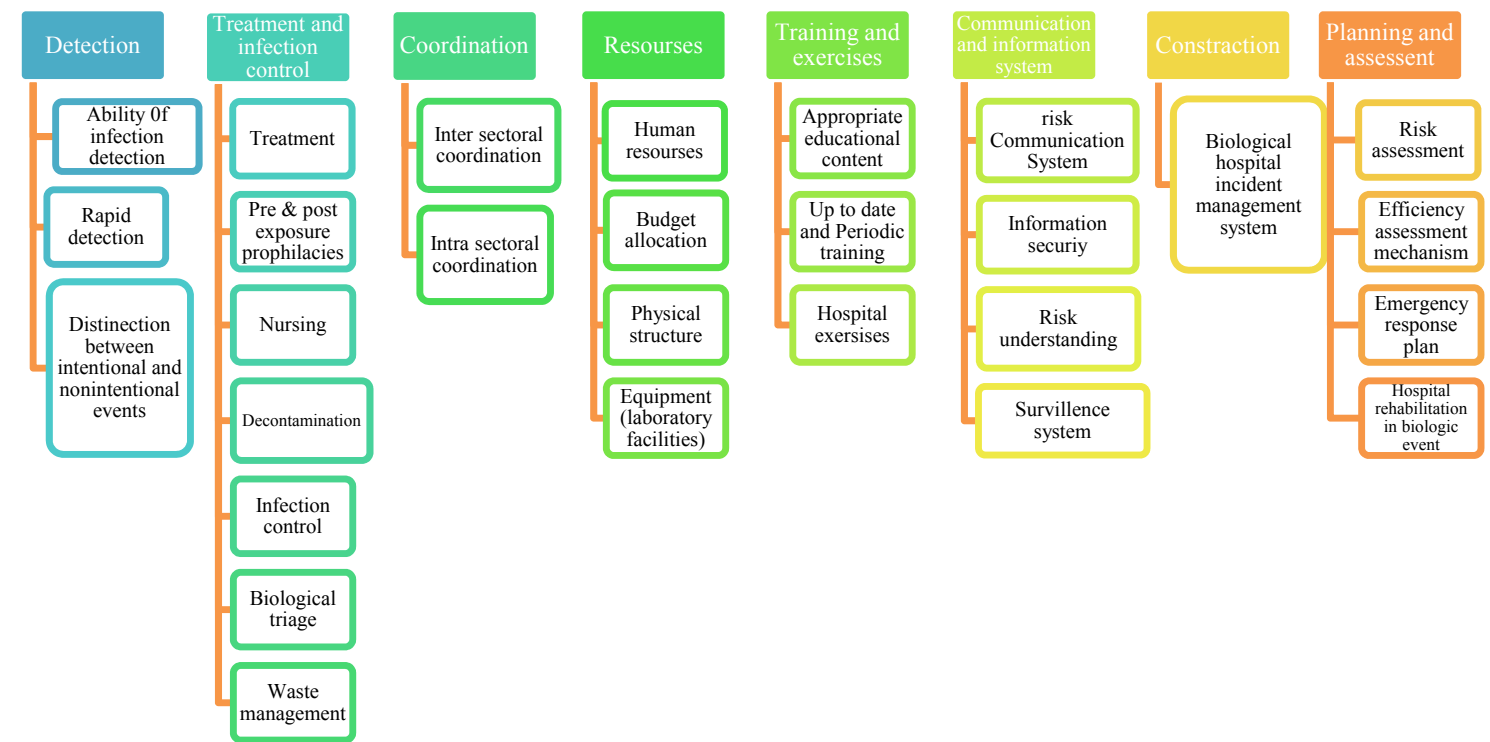

Fig. 1. Extracted categories and subcategories by content analysis

Table 2. Extracted categories and subcategories by content analysis

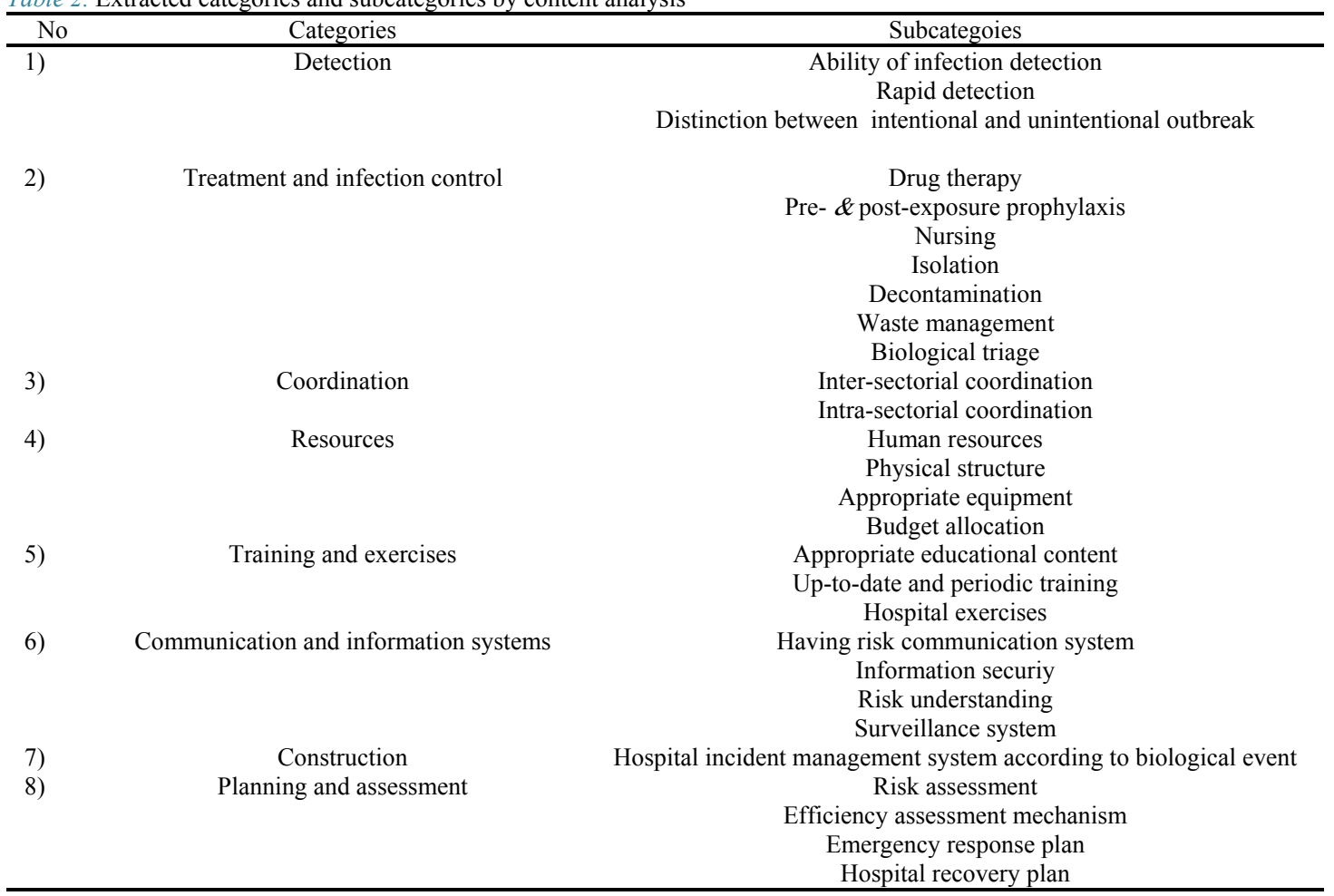

the textbooks, but the bioterrorism detection has not reviewed extensively, and it is very difficult".

\section{Treatment and infection control}

From the participants' perspective, the ability to treat and control the infection in the hospital was considered as an important factor in the proper hospital performance in biological disasters. The obtained subcategories were as follow: the ability to manage the biologic event and treat patients; the ability to care for the patients; the ability to decontaminate the injured people and surfaces; the ability to perform biologic triage; and pre and post exposure prophylaxis in affected individuals.
2-1: Drug therapy of patients and pre and post exposure prophylaxis: The extracted common codes according to participants' interviews were possibility of appropriate response after a biological event in a hospital, prompt and suitable treatment of the patients, having an efficient team of rapid response, the ability to discharge if necessary, pharmaceuticals prescription, having adequate stockpiles, and the ability to perform pre and post exposure prophylaxis.

One participant (P07) said, "If prevention is not imminent with different medications and appropriate vaccines, the disease would spread. For example, if respiratory anthrax or plague becomes prevalent, vaccination and appropriate

\footnotetext{
$4 \quad$ http://mjiri.iums.ac.ir Med J Islam Repub Iran. 2020 (16 Mar); 34:21.
} 
therapeutic prophylaxis are the only way to prevent the disease progress in the community".

2-2: Nursing: The presence of skilled and capable nurses was one of the subcategories obtained in this study. Nurses who are trained, skilled, and familiar with personal protective equipment may have positive effects on hospital performance.

One of the participants (P11) mentioned: "Having skilled nurses to care for infectious patients is valuable as patients' treatment in epidemics. One day I went to the hospital for a visit and saw the AIDS patient wearing a mask. The head-nurse had told him to do so".

2-3: Isolation: According to the participants, the ability to isolate patients with communicable diseases in the hospital and in the designated sites for group isolation was an important component of infection control.

One participant (P05) said, "Isolation spaces are needed based on the causative agent (such as droplet or airborne isolation). In emergency situations, metal partitioning is better, and if not possible, minimal isolation should be executed even with curtains".

2-4 Decontamination: Most participants considered the ability to decontaminate victims efficiently as one of the important factors in hospital performance dealing with biological disasters, which is an important step in decontaminating patients, surfaces, and equipment in proper response to such disasters.

One of the participants (P01) stated, "Environmental health authorities should be active in tackling the issue. Notifications should be declared as soon as possible. Environmental decontamination is needed for each disease".

2-5: Triage: According to participants, the use of specialized triage in biological events affecting many victims will enhance hospital performance. Biological triage which has a different mechanism than other triage systems helps to reduce infection transmission and provide high-speed treatment for people with high-priority care. It also provides the most services in the least amount of time to the largest number of victims.

One participant (P12) said, "All hospitals should have a triage system and special checklists." Biological triage is necessary. There are no triage systems for infectious diseases in my hospital, so there is not any sorting during an outbreak".

2-6: Infection control: Participants considered infection control as an essential criterion for hospital performance in biological disasters. The common codes derived from these subcategories are as follow: the ability to control infections during deliberate or natural biological outbreaks; the availability of preventive drugs at a predetermined time during an epidemic of communicable diseases; appropriate vaccination of people at risk; and the safety of hospitalized or outpatients patients in the outbreak of infectious diseases; and waste management.

One participant (P04) stated, "To see how a hospital performs, we need to see how much patient's safety is considered. Sometimes, with a simple maneuver such as washing hand, transmission of the infection would be prevented".

\section{Coordination}

Participants considered inter-sectoral and intra-sectoral coordination as an important factor in the proper hospital performance during biological disasters.

3-1: Intra-sectorial coordination: A common subcategory was coordination and collaboration within the hospital during a biological disaster, including predetermined inter-organizational tasks.

One participant (P02) said, "When epidemics occur, it is highly important that internal parts of the hospital (such as laboratory, radiology, emergency departments, and others) be coordinated. In the early days of influenza epidemic, we did not know where to send the patients' samples for definite diagnosis, which made the process more complicated".

3-2: Inter-sectorial coordination: The common codes in this subcategory were as follow: Existence of memoranda between hospitals and partner centers (such as organizations for corpses burial, drug production companies, vaccines and personal protective equipment producers, reference laboratories, buildings' owners that can be used for mass storage or isolation).

One of the participants (P10) stated, "If there is a suspicious anthrax case and the hospital is a regional one, they should know which laboratory would help, and there should be an accepted guideline for referral".

\section{Resources}

According to participants' viewpoint, the availability of appropriate human resources, necessary equipment, appropriate physical structure, and enough funding has a beneficial effect on hospital performance in biological disasters. These subcategories created the category of resources.

4-1: Human resources: According to the participants' perspective, one of the most important factors affecting hospital performance was the availability of human resources in biological emergencies in terms of quality and quantity. Defining an organized structure of the staff for the response, duty description for the team, the right staff, and an efficient manager in the hospital seemed necessary at the time of biological disasters.

One participant (P01) said, "Sometimes we have cases of flu, meningitis, and tuberculosis, but the hospital performance is not appropriate and practical. Although training was provided and relative preparedness was expected, during the swine flu outbreak, all staff were afraid and wore masks in 2 hospitals. Even the staff in the infectious diseases ward wore masks, which was not necessary and only caused horror. Only those who are within a meter of the patient should wear a mask".

4.2: Physical structure: Based on participants' viewpoint, appropriate physical structure of hospitals to respond to biological disasters is an effective factor in dealing with biological disasters. The proper physical structure of a hospital, enough space for biologic triage, suitable isolation space considering the type of agent, a proper radiology and laboratory structure, and a standard lab were effective subcategories of this category.

One participant (P03) said, "One of the performance evaluation factors is whether the hospital has been standardized; having a triage space and decontamination room 
before entering to the main ward and isolation from the emergency department are highly important. In the influenza epidemic, all hospitals were ordered to separate the patients upon arrival at hospitals, but this was not possible in some hospitals".

4-3: Equipment: The subcategories were equipping some ambulances ready for a biologic event, having special and practical equipment when dealing with biological disasters, adequate personal protective equipment, and the ability to maintain laboratory biosafety.

One participant (P11) recommended, "Having enough equipment to be able to provide good biosecurity is of significant importance. There is not a special stretcher for contagious patients in our hospital, and the lack of a special stretcher for carrying infected patients can impair performance".

4-4: Budget: According to the participants' opinion, allocation of appropriate budget for hospital was an effective factor in assessing the hospital performance during biological disasters. Insurance of staff and the hospital, and proper allocation of funds were the common subcategories of this category.

One of the participants (P12) said, "My hospital manager is concerned about the financial aspect, and he has limitations on training and recruiting human resources. We do not have financial resource even for equipment and training, and our managers do not believe in spending money for managing these disasters. They prefer to deal with tangible issues rather than intangible issues such as biological disasters that have not yet happened".

\section{Training and exercises}

Training and exercise influence hospital performance. The subcategories were appropriate educational content in accordance with the up-to-date changes in the field and intermittent exercises.

5-1: Educational content: The following subcategories were extracted: Having rich and up-to-date educational content with emphasis on biological group A \& B agents; providing training on how to use personal protective and prevention equipment, triage, isolation, secure area, control, and treatment; and providing training for misbelief correction.

One of the participants (P12) said, "There should be an educational content related to the subject; it can be a part of hospital accreditation. Some employees even do not know the correct pronunciation of diseases".

5-2: Training: All participants considered staff training as one of the most important factors in hospital performance. The extracted subcategory was receive training in the field of biological agents, including continued and regular education, up-to-date training, and training about preventive and personal protective equipment (mask types and time), isolation types, triage, secure area, and group A \& B agents.

One participant (P09) recommended, "Up-to-date training should be available for staff with an interval of maximum of up to 1 year. Our personnel have been graduated many years ago, and their training has not been updated at all".

5-3: Exercise: By analyzing the data, intermittent and proportional exercises were obtained as common codes in the exercise subcategory. According to participants, the factors of this subcategory included the need to conduct a biological exercise in the hospital, and presence of a range of exercises such as top table exercise for managers and coordinators and other types of exercises, and execution of intermittent drills within a specified time schedule.

One of the participants (P11) declared, "Our managers did not have any experience in biological disaster management, even in the form of a drill. We should exercise and learn what we lack".

Another participant (P01) said, "There should be an exercise index for evaluation (drills about personal protective equipment) ".

\section{Communication and information systems}

According to the results of the data analysis, the risk communication system, information security, risk understanding, and surveillance system were subcategories of the communication and information system category.

6-1: Risk communication systems: Having a risk communication system, information security, risk understanding, and a surveillance system were determined as subcategories of this category.

6-2: Information security: Information security is important in an outbreak of infectious diseases in hospitals, as non-patients may go to the hospital for fear of panic or patients may not come to the hospital for fear of stigma. The common codes were as follow: The possibility of sending written reports or internal automation to senior officials, laboratories, and the Ministry of Health, and lack of public notification in some instances.

One of the participants (p10) said, "A false outbreak would occur without proper communication with the economic authorities. Each time after a rumor about an infectious disease, many will come to hospitals as false patients, and controlling such situations would be very difficult".

6-3: Risk understanding: This subcategory was also derived from the information and communication category. Participants believed that developing a positive attitude towards the possibility of biological disasters in hospital managers is crucial, because if a manager or staff does not believe in the possibility of biological disasters, there would be no possibility of necessary and timely action.

One participant (P10) declared, "Hospital managers do not have a proper attitude about this kind of problems. Even after being trained by senior officials, they still do not have a good perception of the biologic disasters".

6-4: Surveillance system: According to participants, the use of surveillance systems in hospitals and the active registration of infectious diseases were considered as effective factors in hospital performance.

One participant (P02) said, "The center for diseases control of Ministry of Health has a tradition of its own. In the health sector, they have a disease expert who will inform you if something happens. They themselves do not actively seek out any disease". 


\section{Construction}

The existence of an appropriate hospital incident command system (HICS) for biological events was one of the main obtained categories in this study. Extracted categories were the need for existence of a code for the biological crisis, type of system activation, existence of a unique command, use of qualified advisors in a commanding system, and proper organization of the staff.

One of the participants (P09) announced, "Having an incident command plan during a biological disaster is highly important. Commanding systems do not work well in our hospitals in important and dangerous disasters such as earthquake and floods".

\section{Planning and assessment}

Planning and assessment were among the other key elements that influenced hospital performance during emergencies. Risk assessment in hospitals was identified as a basic point in evaluation of hospitals. In the absence of a risk assessment in a hospital, finding priorities for risk reduction measures can lead to resources loss and parallel work. Participants considered the factors that impacted hospital performance: the existence of qualified self-assessment mechanisms along with appropriate indicators in assessment checklist and a well-defined cutoff point. Extracted subcategories were existence of updated instructions and guidelines, a special response and recovery plan for biologic threats, using the all-hazards approach to preparedness, and a lab plan.

Another participant (P06) said, "Readiness assessment checklist must be completed every 3-4 months for each center. Our hospitals do not have any assessment checklist for infectious diseases".

\section{Discussion}

In this qualitative study, which was done using content analysis, the effective factors for hospital performance in biological emergencies in IR of Iran were identified as follow: diagnosis; treatment and control of infection; resources; coordination; training and practice; communication and information systems; construction; and planning and assessment.

\section{Detection}

Detecting a biologic event is one of the primary influencing factors in hospital performance. Early diagnosis is one of the important factors for initiating immediate action and response to prevent further development of a biological agent (26). Controlling hospital outbreaks requires rapid diagnosis and search for clusters; then, appropriate controls are executed (27). Early diagnosis of the disease prevents its spread and can be effective in the timely treatment of exposed individuals and doing biologic triage. While an epidemic is rapidly discovered, its spread can be prevented by isolating patients and prompt prophylaxis. Also, recognizing the type of biological disaster (intentional or unintentional) aspect may have an impact on hospital function because management of such biological emergencies may en- counter many complications considering type of agent, genomic manipulation, event location, geological and climatic conditions, and disease spread in the community prior to definitive diagnosis (28). Over the past 2 decades, the intentional enhancement of using biological agents has increased the demand for risk assessment and monitoring of such events, which often involves modeling approaches based on certain assumptions such as the ability to generate, store, and distribute. The ability to release a biological agent as a weapon does not only result in the creation of airborne transmissible microorganisms but can be transmitted from human to human and spread. Management of deliberate transmissible is different from that of non-transmissible epidemics (26).

\section{Treatment and infection control}

Another effective factor in hospital performance in biological events is treatment and infection control. In a biologic emergency, treatment measures and timely response to the event are important factors. Triage is an important factor in controlling hospital infection during a biological emergency (29). Most triage systems deal with traumatic or kinetic injuries (30). Such systems are not applicable to other types of disasters, including biologic emergencies, because some factors (eg, exposure and symptoms) do not affect the infection control and make rapid diagnosis and treatment more difficult. Victims are unlikely to be harmed, and there may not be a particular scene of disaster (31). Therefore, it is necessary to consider a special system of triage instead of conventional systems (30).

In accordance with Burkle's recommended method, biologic triage is used for patients in an incident with a large number of injured people and divides them into 5 groups: (1) susceptible but not exposed; (2) exposed but not yet infectious; (3) infectious; (4) removed by death or recovery; and (5) protected by vaccination or prophylactic medication (30). In settings where infectious diseases are easily transmitted, deaths from infectious diseases are more likely than traumatic events (32). Therefore, applying biologic triage is vital for controlling transmission. During biologic emergencies, triage makes it easier to control and treat the patients, prevents loss of resources, and reduces the probability of transmission of communicable diseases, and decreases the burden of hospitalization by reducing the number of visits to hospitals $(29,32)$. In this regard, access to drug and vaccine supplies and appropriate measures can reduce the number of patients or decrease the disease severity. Effective actions in performance are hand washing and self-protection methods for staff, and proper isolation based on the type of disease $(33,34)$. Providing care for patients within the hospital is an important part of patients' treatment and infection control at treatment centers (35). Therefore, having competent nurses and trained infection control specialists as well as self-protection methods are other measures related to performance improvement (36-40). In biologic emergencies, one means of differentiation with other common emergencies is the need for self-protection methods of staff, especially physicians and nurses who have close contact with the patients $(29,41)$. Taking appro- 
priate measures stops the transmission of the infection between patients and staff, including nurses $(29,34,35,42-$ 47). Decontamination spaces of biological patients will make these disasters distinct from others. Decontamination is less important in disasters with large number of traumatic patients, but in biological disasters (especially man-made), the entry of noncontaminated patients into hospitals and the implementation of individualized and collective quarantine are of great importance $(29,41,48)$. Risk management approaches for infectious disasters are necessary to reduce the risk of secondary contamination with regards to decontamination measures and surveillance (26).

\section{Resources}

Based on the results of this study, human and financial resources, physical structures of hospitals, and equipment (including personal protective equipment, laboratory, appropriate vaccine, and antibiotics) are effective factors in the proper functioning of hospitals. These findings were consistent with those of other studies $(29,49-52)$. An appropriate response requires access to laboratory facilities (53). Decontamination facilities and access to personal protective equipment for triage and decontamination teams are among the limitations of performance in biological events $(4,41,54)$. Adequate budget is usually not allocated due to the high cost of preparedness and performance measures in infectious disasters. Several studies have shown that the number of public health staff may decrease at the time of biological disasters (such as flu pandemics) (55-57). Typically, volunteers will meet the required human resources (efficient and trained personnel) in disasters $(58,59)$; however, this would not be the case in biological disasters. Although the amount of motivation to work during biological disasters varies from country to country, the total amount of motivation is lower than in other events $(29,56)$. This drop in staff motivation is also evident in the number of volunteers (29). However, more research is needed to study the willingness of public health staff in disasters (20). Therefore, one of the most important factors in biological disasters is the provision and management of manpower required in hospitals. Previous studies have shown that male gender, being a physician, having a full-time job, self-protection, and communication equipment for staff, and basic needs such as water have a positive impact on the willingness to work in such events $(55,60,61)$. Motivation facilitators for working in infectious emergencies include access to vaccination and personal protective equipment, flexible work shift, taking care of staff children, and information sharing (57).

Regarding the release of a biologic agent, the strategic storage of the vaccines as well as pharmaceuticals for treatment of the agent can be important because easy access to antibiotics and vaccines is very effective. Access to ventilators for the management of infectious respiratory disasters is a necessity $(61,62)$. There are currently antiviral drug storages for responding epidemics that cost a lot (63). The lack of funding and financial resources is a major obstacle to the preparedness and proper performance in infectious disasters $(64,65)$.

Appropriate supply strategies, adaptation to the severity of the event, and the type of microorganism have a preventive and controlling role in infection from person to person $(26,66)$. Also, having a proper physical structure to respond to biological events is important for proper functioning. This factor has been mentioned in numerous articles (67-70). Examples which may be presented here are the existence of a separate entrance door for the emergency department, proper design of the rooms and the hospital, and proper equipment (eg, separate ventilators in the emergency department) (69).

\section{Education}

Based on the findings of this study, staff training was one of the main elements of the proper functioning of hospitals in biological events. Reviewing articles also indicated that education and training are key elements in disaster preparedness $(3,71)$. Many efforts have been made to explain the capabilities and design of training curricula for management and response to CBRNE events, but there is still lack of capability-based plans (3). Emergency department physicians, nurses, and support staff are the 3 main groups for training and education. Hospitals will not be able to respond appropriately in disasters without the upgraded educational guides (71). Training is an important challenge in managing disasters which was obvious in events such as Ebola outbreak. Control of communicable diseases such as Ebola and other infections may be affected by lack of educational materials, curricula (educational curriculum, development of educational contents, training resources, and tools), and educational contents (72). Most of training courses (during and after Ebola epidemic) have been performed for infection control staff and has not been addressed for other stakeholders (65). Best practices for Ebola education are engaging all stakeholders (eg, crisis managers, infectious disease control staff, and health workers) in educational programs. The most important educational challenges are annual budget and misdeclaration of sufficient training in an organization $(65,73)$. Sustained education for combating the spread of infectious diseases requires annual budget, full support of the organization management, and engaging all stakeholders (65). The reports show poor knowledge about disaster planning and biological events in emergency departments around the world (34, $71,74)$. It is imperative to ensure full recognition of risk reduction plans in infectious disasters for all those responsible for reducing the risk. In an Egyptian study, medical residents had less training on personal protective equipment than specialists and counselors (73). However, education in this regard is one of the key points in infection transmission and control. Occupational and non-occupational stresses in physicians were more than nurses, indicating the need for further training on the nature of pandemics, the results, complications, and methods of infection prevention (73, 75). In the absence of comprehensive support and failure to address the motivation or needs of professionals, effective education and the use of educational opportunities would be a challenge (76). Developing educational standards and guidelines for a medical response to disasters (especially CBRN) has a major impact on emergency response to disasters (65). Some requirements are appropriate educational 
content, determining the type of training, and evaluation of tools. A review article of CBRNE training courses between 2006-2016 indicated that course evaluation was not done by any study (77). Essential elements of education are personal protective training, hospital incident commanding systems for emergencies, surge capacity, and assessment and risk determination in accordance with biological disasters (3).

\section{Coordination}

Coordination is one of the disaster management requirements in communities and hospitals. Disaster management occurs in a complex context. This complexity is the result of a variety of different functions of the external and internal sectors in a hospital. Coordination of these sectors would result in proper disaster management. In this study, internal and external coordination were key factors in response to biologic disasters. The presence of the emergency coordinator promotes health sector preparation activities. Coordinators also provide a road map for moving in and out of hospital-crisis-related factors. Avery et al indicated that the presence of a coordinator increases the level of readiness in the health sector in a disaster (78). This is evident especially in infectious disasters which require more coordination between infectious disease control centers and security sectors. Jones et al showed that the coordinator had a direct relationship with the readiness of hospitals in pandemics. The connection was not linked to readiness in other settings such as casualty incidents, general preparedness of the hospital, and inefficiency of the hospital infrastructure. This could be a sign of the difference in infectious disasters. Management requirements of such events include provision of vaccines, drugs, and personal protective equipment, and sufficient equipment and personnel resources at local, national, and international levels. Memoranda of agreements (MOAs), memoranda of understanding (MOUs), and planning partnerships with other hospitals, health centers, government, local authorities, and other providers of support services are examples of out of organization coordination (78). Capability of hospital external evacuation also requires external organization coordination, which according to the review of articles in the field of interhospital memoranda, has the lowest rate for children and infants (79).

\section{Communications and information systems}

Risk communication is one of the central components of proper performance of hospitals during infectious disasters (80). Communications and information systems were derived as one of the main categories in this study. When an outbreak occurs and general public health is compromised, direct interventions and treatment options may be limited due to lack of time and the need for resources. Therefore, communication, notification, and guidance are often the most important tools of public health in risk management during such events (80). Communication readiness reduces the response time of the crisis (80). Other impacts of communication and information systems during disasters are confidence of people in managers and acceptance of protective behaviors, disease surveillance, and reduction of confusion (80). To effectively respond, information should be organized in a timely fashion and be disseminated through multiple channels along with appropriate training (81). Health communication includes 4 key elements: message, receiver, source of information, and the channel for information exchange. The above elements should exist and function properly in any health-related communication plan. Communication would be implemented in the field of informing the public as well as in health systems, especially with respect to infectious disasters (82). Public health stakeholders, although not directly involved in public health emergency management, will take timely decisions, plan, and control timely access to information (80). Moreover, to effectively implement communication in these disasters, standardized educational content, clear national guidelines, and pre-prepared plans in the field of communication in hospitals are required (81). Ineffective communication is one of the potential reasons for the failure of infection control in health workers (83). Hospital communications should be completely clear, scientific, and understandable. Health workers are an important criterion for community's trust in resources and their knowledge and dealing with the situation will affect the trust of the community (80). Bonneux et al suggest that the management of unpredictable panic of hospital staff is more difficult than controlling the spread of disease (84). The clear release of information can prevent the fear of staff and society (19), and a long negative impact will occur with no on time intervention on hospital performance (80). Understanding the proper risk from leading risk is a decisive factor in disaster management. Today, in a world where the information transfer (whether right or wrong) is done at a rapid pace, the perception of risk from truly risky cases is less than estimated, and the risk of rare cases is overestimated (84). Understanding the risk is related to the mental sensation of control. The threat of an epidemic may in part be frightening because of feeling lack of control that leads to an unwanted activity (84).

Verbal communication and standard communications (eg, guidelines, education, electronic communications, and marketing) are definitive communication pathways in infectious disasters. Experiences indicate that traditional methods are unsuccessful in changing and maintaining the best performance in infection control, although newer methods (such as electronic communications and marketing) have some problems. Some approaches (eg, involving health staff in the communication processes or up-down communication) can improve communication methods (63). The timely identification of cases of infectious diseases with increased patients over a given period of time is a critical necessity for the IPCs. Survival systems in hospitals are currently focusing on a small set of microorganisms such as methicillin-resistant staphylococcus aureus, Carbapenem-resistant enterobacteriaceae, and clostridium difficile (27). There are several pathways for the transmission of pathogens (staff to patient, environment to patient, patient to patient, visitors to patient, etc.) due to dynamic health care in hospitals. Better monitoring of the epidemics onset would be implemented by operating the modern and multifunctional surveillance systems, including observing 
symptom reports in specific time spots, syndromic surveillance systems, prediagnosis and nonspecific criteria monitoring, and health behaviors observation (eg, absenteeism from work or school, pharmacy referral or even search rate in search engines with specific words that indicate an increase in the incidence of infectious diseases) (28). Although more modern systems such as electronic surveillance systems have high sensitivity, their positive predictive value is low (27). United States has conducted surveillance systems and epidemiological surveillance with a large budget in the framework of BioWatch program to monitor the deliberate propagation of biological agents (26).

\section{Construction}

In this study, an incident management commanding structure in accordance with biological emergencies was found to be the proper structure of the main element of performance. Hospital incident command systems are used as a model for disaster response in some countries. This system is an attempt for standard performance of hospitals in disasters $(85,86)$. In recent years, incident command system has been implemented for Iranian hospitals to manage disasters with an all-hazards approach (87). Application of this system is part of the requirements for the accreditation of hospitals in IR of Iran. However, this system has limitations such as not addressing the vulnerability of hospitals and not assessing the hospital performance in disasters (86). The participants considered the incident command system (consistent with biological events) as an effective element of hospital performance in biological events. Use of the incident command system is part of an appropriate response to CBRN events $(88,89)$. The hospital incident commanding system is an integrated structure that despite the volume and effects of the events can provide coordination, control, operations, planning, support, and other necessary functions for event management, if properly implemented. It also explains responsibilities clearly leading to appropriate response (90). The effectiveness of this system has been indicated in outbreaks of infectious diseases $(91,92)$. If the response to biological emergencies will be designed as a systematic approach, it would lead to faster response by establishing coordination, speed of communication, recalling staff, etc. (29). This adaptation could include selection of specialized infectious and epidemiologic consultants, proper planning, operations based on infection control requirements, treatment and care, and other requirements for managing biological crises. In addition, matching these systems with biological emergencies can speed up the response by increasing external and intra-organizational coordination of multiagent organs (91).

\section{Planning and assessment}

Planning was one of our extracted main categories. The existence of disaster management plans in hospitals is essential to ensure preparedness and response, even before the emergence of events. A disaster management plan is a set of procedures, policies, interactional patterns, roles, and contingencies which are developed to prepare and implement appropriate response to a crisis (9).
This plan includes staff training plans, responding to potential biologic agents, pollution prevention, rapid communication plans, potential quarantine exposure (individual and group), resource production and planning, and rapid diagnostic plans $(26,48)$. The existence of evidence-based guidelines is one of the deficiencies in infection control in Iranian hospitals. Self-assessment, external evaluation, and the use of intra-organizational and external experts for scientific review of guidelines and plans can have a positive effect on improving hospital performance. However, performance evaluation can be assessed in real terms after disasters and from lessons learned (1).

Security issues affecting the health and national systems and context of intentional biologic events were limitations of the present study, which did not allow all selected managers to participate in the study. However, the limitation was resolved by continuing the interviews with the main managers, determining the alternative ones, and continuing the interviews until saturation.

\section{Conclusion}

Hospital management in outbreaks of infectious diseases (intentional or unintentional) is complex and requires different planning than natural disasters such as earthquakes, floods, etc. In such disasters, readiness to respond and appropriate action is a multifaceted operation that has not been addressed in IR of Iran and other countries so far. In this study, the factors affecting performance in such events were qualitatively explained and categorized. Also, to properly perform in such disasters, each of the 8 categories and their subcategories should be carefully implemented with detail.

Developing a model for assessing hospitals' performance in biological events should be considered in future studies. The researchers also recommend developing tools for evaluating hospitals performance and preparedness for biological disasters. Practical suggestions include testing the efficacy of extracted elements of this study in hospital drills, full-scale practices, and actual hospital events.

\section{Acknowledgements}

We would like to thank all of participants who spent their valuable time for interviews and other surveys.

\section{Conflict of Interests}

All authors disclose that there is not any actual or potential conflict of interest, including any financial, personal, or other relationships with relevant authorities or organizations within 3 years of starting the study.

\section{References}

1. Droogers M, Ciotti M, Kreidl P, Melidou A, Penttinen P, Sellwood C, et al. European Pandemic Influenza Preparedness Planning: A Review of National Plans, July 2016. Disaster Med Public. 2018:1-11.

2. Ide R, Hegermann-Lindencrone M, Sayohat Hasanova DP, Dolyan N, Kasymbekova K, Abdulakhad, et al. Innovative Pandemic influenza preparedness Framework paves the way for sustainable improvements to pandemic preparedness. Public Health Panorama. 2018;4(1):78-84.

3. Djalali A, Della Corte F, Segond F, Metzger MH, Gabilly L, Grieger F, et al. TIER competency-based training course for the first receivers of CBRN casualties: a European perspective. . Eur J Emerg Med. 
2017;24(5):371-376.

4. Mortelmans LJM, Gaakeer MI, Dieltiens G, Anseeuw K, Sabbe MB Are Dutch Hospitals Prepared for Chemical, Biological, or Radionuclear Incidents? A Survey Study. Prehosp Disaster Med. 2017;32(5):483-91.

5. Green MS, LeDuc J, Cohen D, Franz DR. Confronting the threat of bioterrorism: realities, challenges, and defensive strategies. Lancet Infect Dis. 2019;19(1):e2-e13.

6. Balali-Mood M, Moshiri M, Etemad L. Medical aspects of bioterrorism. Toxicon. 2013;69:131-42.

7. Narayanan N, Lacy CR, Cruz JE, Nahass M, Karp J, Barone JA, et al. Disaster Preparedness: Biological Threats and Treatment Options. Pharmacotherapy. 2018;38(2):217-34.

8. Center for systematic peace. Global conflict trends. Available http://www.systemicpeace.org/conflicttrends.html.; 2016 [cited Accessed 7 February 2019].

9. Olivieri C, Ingrassia PL, Della Corte F, Carenzo L, Sapori JM, Gabilly $\mathrm{L}$, et al. Hospital preparedness and response in CBRN emergencies: TIER assessment tool. Eur J Emerg Med. 2017;24(5):366-70.

10. Razak S, Hignett S, Barnes J. Emergency Department Response to Chemical, Biological, Radiological, Nuclear, and Explosive Events: A Systematic Review. Prehosp Disaster Med. 2018;33(5):543-9.

11. Forestier C, Cox AT, Horne S. Coordination and relationships between organisations during the civil-military international response against Ebola in Sierra Leone: an observational discussion. J R Army Med Corps. 2016;162(3):156-62.

12. Chan YF, Alagappan K, Gandhi A, Donovan C, Tewari M, Zaets SB. Disaster management following the Chi-Chi earthquake in Taiwan. Prehosp Disaster Med. 2006;21(3):196-202.

13. De Montravel G. Revue du Corps de sante colonial. Med Tropic. 2002;62(4):445-52.

14. Borio L, Inglesby T, Peters CJ, Schmaljohn AL, Hughes JM, Jahrling $\mathrm{PB}$, et al. Hemorrhagic fever viruses as biological weapons: medical and public health management. JAMA. 2002;287(18):2391-405.

15. Veillard J, Champagne F, Klazinga N, Kazandjian V, Arah OA, Guisset AL. A performance assessment framework for hospitals: the WHO regional office for Europe PATH project. Int J Qual Health Care. 2005; 17(6):487-96.

16. Groene O, Klazinga N, Kazandjian, Lombrail P, Bartels P. The World Health Organization Performance Assessment Tool for Quality Improvement in Hospitals (PATH): An Analysis of the Pilot Implementation in 37 Hospitals. Int $\mathrm{J}$ Qual Health Care. 2008;20(3):155-61.

17. Djalali A, Ragazzoni L. Role of Hospitals in a Disaster. Ciottone's Disaster Medicine. 2nd ed. USA2015.

18. Kaji AH, Langford V, Lewis RJ. Assessing hospital disaster preparedness: a comparison of an on-site survey, directly observed drill performance, and video analysis of teamwork. Ann Emerg Med. 2008;52(3):195-201, .e1-12.

19. Kotora JG. An assessment of Chemical, Biological, Radiologic, Nuclear, and Explosive preparedness among emergency department healthcare providers in an inner city emergency department. Am J Disaster Med. 2015;10(3):189-204.

20. Gowing JR, Walker KN, Elmer SL, Cummings EA. Disaster Preparedness among Health Professionals and Support Staff: What is Effective? An Integrative Literature Review. Prehosp Disaster Med. 2017;32(3):321-8

21. Lane JE, Dimick J, Syrax M, Bhandary M, Rudy BS. Bioterrorism and disaster preparedness among medical specialties. Am J Disaster Med. 2012;7(1):48-60.

22. Baucher MA, Kearns P, Gyenes Z, Heraty W, Baubion C, Gamper C, et al. Towards an All-Hazards Approach to Emergency Preparedness and Response. Lessons Learnt from Non-Nuclear Events. Organisation for Economic Co-Operation and Development; 2018.

23. Graneheim UH, Lundman B. Qualitative content analysis in nursing research: concepts, procedures and measures to achieve trustworthiness. Nurse Educ Today. 2004;24(2):105-12.

24. Liang SY, Chuang YH, Wu SF. Preliminarily application of content analysis to qualitative nursing data. Hu Li Za Zhi. 2012;59(5):84-90.

25. Campos CJ, Turato ER. Content analysis in studies using the clinicalqualitative method: application and perspectives. Rev Lat-Am Enferm. 2009; 17(2):259-64.

26. Grundmann O. The current state of bioterrorist attack surveillance and preparedness in the US. Risk Manag Healthc Policy. 2014;7:177-87.

27. Stachel A, Pinto G, Stelling J, Fulmer Y, Shopsin B, Inglima K, et al.
Implementation and evaluation of an automated surveillance system to detect hospital outbreak. Am J Infect Control. 2017;45(12):1372-1377

28. Kman NE, Bachmann DJ. Biosurveillance: A Review and Update Adv Prev Med. 2012;(2012):1-9

29. Geiling J, Burkle FM. Mass Casualty Incidents: Organizational and Triage Management Issues. In Civetta, Taylor \& Kirby's: Critical Care, 5th Edition, Wolters Kluwer 2018.

30. Christopher A, Kahn EBL, Cone DC. TRIAGE. In: Kristi L. Koenig CHS, editor. Koenig and Schultz's Disaster Medicine Comprehensive Principles and Practices 2nd Edition. USA2016. p. 174-81.

31. Cone DC, Koenig KL. Mass casualty triage in the chemical, biological, radiological, or nuclear environment. Eur J Emerg Med. 2005;12(6):287-302.

32. Burkle FM. Triage and the Lost Art of Decoding Vital Signs: Restoring Physiologically Based Triage Skills in Complex Humanitarian Emergencies. Disaster Med Public. 2018;12(1):76-85.

33. Munoz-Price LS, Arheart KL, Mills JP, Cleary T, Depascale D, Jimenez A, et al. Associations between bacterial contamination of health care workers' hands and contamination of white coats and scrubs. Am J Infect Control. 2012;40(9):e245-e8.

34. Carlos C, Capistrano R, Tobora CF, delos Reyes MR, Lupisan S, Corpuz A, et al. Hospital preparedness for Ebola virus disease: a training course in the Philippines. Western Pac Surveill Response J. 2015;6(1):33-43

35. Jabbari H, Alikhah H, Alamdari NS, Behzad MN, Mehrabi E, Borzu $\mathrm{L}$, et al. Developing the Use of Quality Indicators in Sterilization Practices. Iran J Public Health. 2012;41(7):64-9.

36. Musau J, Baumann A, Kolotylo C, O'Shea T, Bialachowski A. Infectious disease outbreaks and increased complexity of care. Int Nurs Rev. 2015;62(3):404-11.

37. McMullan C, Brown GD, O'Sullivan D. Preparing to respond: Irish nurses' perceptions of preparedness for an influenza pandemic. Int Emerg Nurs. 2016;26:3-7.

38. Pintar PA. An intrepreneurial innovative role: integration of the clinical nurse specialist and infection prevention professional. Clin Nurse Spec. 2013;27(3):123-7.

39. Smith C, Hewison A. Are nurses prepared to respond to a bioterrorist attack: a narrative synthesis. J Adv Nurs. 2012;68(12):2597-609.

40. Summers A. Pandemic flu: lessons from the Toronto SARS outbreak. Emerg Nurse. 2009;17(5):16-9.

41. Daugherty EL. Health care worker protection in mass casualty respiratory failure: infection control, decontamination, and personal protective equipment. Respir Care. 2008;53(2):201-12; discussion 12 14.

42. Raboud J, Shigayeva A, McGeer A, Bontovics E, Chapman M, Gravel D, et al. Risk Factors for SARS Transmission from Patients Requiring Intubation: A Multicentre Investigation in Toronto, Canada. Plos One. 2010;5(5):10.

43. Chu TP, Li CC, Wang L, Hsu LW, Eng HL, You HL, et al. A surveillance system to reduce transmission of pandemic H1N1 (2009) influenza in a 2600-bed medical center. PLoS One. 2012;7(3):e32731.

44. Du M, Suo J, Jia N, Xing Y, Xie L, Liu Y. The cross-transmission of 2009 pandemic influenza A (H1N1) infections among healthcare workers and inpatients in a chinese tertiary hospital. Infect Control Hosp Epidemiol. 2012;33(3):295-8

45. Killingley B. Investigations into human influenza transmission. Ph.D.dissertation. Ann Arbor: The University of Nottingham (United Kingdom); 2013.

46. Balkhy HH, Perl TM, Arabi YM. Preventing healthcare-associated transmission of the Middle East Respiratory Syndrome (MERS): Our Achilles heel. J Infect Public Heal. 2016;9(3):208-12.

47. Blanco N, Eisenberg MC, Stillwell T, Foxman B. What Transmission Precautions Best Control Influenza Spread in a Hospital? Am J Epidemiol. 2016;183(11):1045-54.

48. Li Y, Hsu EB, Links JM. Healthcare system cost evaluation of antiviral stockpiling for pandemic influenza preparedness. Biosecur Bioterror 2010;8(2):119-28.

49. Kang J, O'Donnell JM, Colaianne B, Bircher N, Ren D, Smith KJ. Use of personal protective equipment among health care personnel: Results of clinical observations and simulations. Am J Infect Control. 2017;45(1):17-23.

50. Phin NF, Rylands AJ, Allan J, Edwards C, Enstone JE, Nguyen-VanTam JS. Personal protective equipment in an influenza pandemic: a UK simulation exercise. J Hosp Infect. 2009;71(1):15-21.

51. Mitchell R, Ogunremi T, Astrakianakis G, Bryce E, Gervais R, Gravel 
D, et al. Impact of the 2009 influenza A (H1N1) pandemic on Canadian health care workers: A survey on vaccination, illness, absenteeism, and personal protective equipment. Am J Infect Control. 2012;40(7):611-6.

52. Mitchell R, Roth V, Gravel D, Astrakianakis G, Bryce E, Forgie S, et al. Are health care workers protected? An observational study of selection and removal of personal protective equipment in Canadian acute care hospitals. Am J Infect Control. 2013;41(3):240-4.

53. Khan AS, Lurie N. Health security in 2014: building on preparedness knowledge for emerging health threats. Lancet. 2014;384(9937):93-7.

54. Meltzer MI, Gambhir M, Atkins CY, Swerdlow DL. Standardizing scenarios to assess the need to respond to an influenza pandemic. Clin Infect Dis. 2015;60 Suppl 1:S1-8.

55. Aoyagi Y, Beck CR, Dingwall R, Nguyen-Van-Tam JS. Healthcare workers' willingness to work during an influenza pandemic: a systematic review and meta-analysis. Influenza Other Respir Viruses. 2015;9(3):120-30.

56. Balicer RD, Barnett DJ, Thompson CB, Hsu EB, Catlett CL, Watson $\mathrm{CM}$, et al. Characterizing hospital workers' willingness to report to duty in an influenza pandemic through threat- and efficacy-based assessment. BMC Public Health. 2010;10:436.

57. Rutkow L, Paul A, Taylor HA, Barnett DJ. Perceived Facilitators and Barriers to Local Health Department Workers' Participation in Infectious Disease Emergency Responses. J Public Health Manag Pract. 2017;23(6):644-50.

58. Whittaker J, McLennan, B., \& Handmer, J. A review of informal volunteerism in emergencies and disasters: Definition, opportunities and challenges. Int J Disast Risk Re. 2015;13:358-68.

59. Aliyari S, Zareian, A., Hatami, Z., Aliyari Shorehdeli, M. . Spiritual Experiences and Memories of Healthcare Personnel during the Holy Defense: A Summative Content Analysis. J Milit Med. 2015;17(3):171-

60. Zoutman DE, Ford BD, Melinyshyn M, Schwartz B. The pandemic influenza planning process in Ontario acute care hospitals. Am J Infect Control. 2010;38(1):3-8.

61. Huang HC, Araz OM, Morton DP, Johnson GP, Damien P, Clements $\mathrm{B}$, et al. Stockpiling Ventilators for Influenza Pandemics. Emerg Infect Dis. 2017;23(6):914-21.

62. Meltzer MI, Patel A, Ajao A, Nystrom SV, Koonin LM. Estimates of the demand for mechanical ventilation in the United States during an influenza pandemic. Clin Infect Dis. 2015;60 Suppl 1:S52-7.

63. Edwards R, Sevdalis N, Vincent C, Holmes A. Communication strategies in acute health care: evaluation within the context of infection prevention and control. J Hosp Infect. 2012;82(1):25-9.

64. Mortelmans LJ, Van Boxstael S, De Cauwer HG, Sabbe MB. Preparedness of Belgian civil hospitals for chemical, biological, radiation, and nuclear incidents: are we there yet? Eur J Emerg Med. 2014;21(4):296-300

65. Yeskey K, Hughes J, Galluzzo B, Jaitly N, Remington J, Weinstock D, et al. Ebola Virus Training: A Needs Assessment and Gap Analysis. Health Secur. 2017;15(3):225-9.

66. Porru S, Campagna M, Arici C, Fostinelli J, Tonozzi B, Placidi D. [Vaccinations for health care workers exposed to biohazard. Evidences and good medical practices]. G Ital Med lav Ergon. 2010;32(4 Suppl):298-301

67. Wilson AP, Ridgway GL. Reducing hospital-acquired infection by design: the new University College London Hospital. The J Hosp Infect. 2006;62(3):264-9.

68. Lenaghan PA, Schwedhelm M. Nebraska biocontainment unit design and operations. J Nurs Adm. 2015;45(6):298-301.

69. Bataille J, Brouqui P. Building an Intelligent Hospital to Fight Contagion. Clin Infect Dis. 2017;65(suppl 1):S4-s11.

70. Stockley JM, Constantine CE, Orr KE. Building new hospitals: a UK infection control perspective. J Hosp Infect. 2006;62(3):285-99.

71. Reilly M, Markenson DS. Education and training of hospital workers: who are essential personnel during a disaster? Prehosp Disaster Med. 2009;24(3):239-45.

72. Kratochvil CJ, Evans L, Ribner BS, Lowe JJ, Harvey MC, Hunt RC, et al. The National Ebola Training and Education Center: Preparing the United States for Ebola and Other Special Pathogens. Health Secur. 2017;15(3):253-60.

73. El Gaafary MM, Abd Elaziz KM, Abdel-Rahman AG, Allam MF. Concerns, perceived impacts and preparedness of health care workers in a referral hospital in Egypt in facing influenza (H1N1) epidemic. J Prev Med Hyg. 2010;51(3):105-9.

74. Paganini M, Borrelli F, Cattani J, Ragazzoni L, Djalali A, Carenzo L, et al. Assessment of disaster preparedness among emergency departments in Italian hospitals: a cautious warning for disaster risk reduction and management capacity. Scand J Trauma Resusc Emerg Med. 2016;24(1):101

75. Adelaine SA, Shoaf K, Harvey C. An Assessment of Collaboration and Disasters: A Hospital Perspective. Prehosp Disaster med. 2016;31(2):121-5.

76. Walsh L, Craddock H, Gulley K, Strauss-Riggs K, Schor KW. Building health care system capacity: training health care professionals in disaster preparedness health care coalitions. Prehosp Disaster med. 2015;30(2):123-30.

77. Kako M, Hammad K, Mitani S, Arbon P. Existing Approaches to Chemical, Biological, Radiological, and Nuclear (CBRN) Education and Training for Health Professionals: Findings from an Integrative Literature Review. Prehosp Disaster med. 2018;33(2):182-90.

78. George H. Avery, Zabriskie J. The Impact of Federal Bioterrorism Funding Programs on Local Health Department Preparedness Activities. Eval Health Prof. 2009;32(2):95-127.

79. World Health Organization. Outbreak Communication Planning Guide Switzerland: WHO; 2008.

80. Mosquera M, Melendez V, Latasa P. Handling Europe's first Ebola case: internal hospital communication experience. Am J Infect Control. 2015;43(4):368-9.

81. Locatelli SM, LaVela SL, Hogan TP, Kerr AN, Weaver FM. Communication and information sharing at VA facilities during the 2009 novel H1N1 influenza pandemic. Am J Infect Control. 2012;40(7):622-6.

82. Bartlett JG. Planning for avian influenza. Ann Intern Med. 2006; 145(2):141-4

83. Ong MS, Magrabi F, Post J, Morris S, Westbrook J, Wobcke W, et al. Communication interventions to improve adherence to infection control precautions: a randomised crossover trial. BMC Infect Dis. 2013;13:72.

84. Bonneux L, Van Damme W. An iatrogenic pandemic of panic. BMJ. 2006;332(7544):786-8

85. Djalali A, Castren M, Hosseinijenab V, Khatib M, Ohlen G, Kurland L. Hospital Incident Command System (HICS) performance in Iran; decision making during disasters. Scand J Trauma Resusc Emerg Med. 2012;20:14.

86. Shooshtari S, Tofighi S, Abbasi S. Benefits, barriers, and limitations on the use of Hospital Incident Command System. J Res Med Sci. 2017;22:36.

87. Djalali A, Hosseinijenab V, Peyravi M, Nekoei-Moghadam M, Hosseini B, Schoenthal L, et al. The hospital incident command system: modified model for hospitals in iran. PLoS Curr. 2015;7.

88. Elham Anbari, Mohammad Hossein Yarmohammadian, Isfahani MN. From investigation of hospital protocols and guidelines to designing a generic protocol for responding to chemical, biological, radiological, and nuclear incidents. Int $\mathrm{J}$ Health Syst Disaster Manage. 2015;3(4):195-9.

89. Powers R. Organization of a hospital-based victim decontamination plan using the incident command structure. Disaster Manag Response. 2007;5(4):119-23.

90. Schoonover H, Haydon K. Incident command structure using a daily management system and the Centers for Disease Control and Prevention's Patient Notification Toolkit drives effective response to an infection control breach. J Health Risk Manag. 2018;38(2):19-26.

91. Quinn E, Johnstone T, Najjar Z, Cains T, Tan G, Huhtinen E, et al. Lessons Learned From Implementing an Incident Command System During a Local Multiagency Response to a Legionnaires' Disease Cluster in Sydney, NSW. Disaster Med Public Health Prep. 2018;12(4):539-42.

92. Jacoby IJ. SARS and the Hospital Emergency Incident Command System (HEICS): outbreak management as the mother of invention. J Emerg Med. 2005;28(2):225-6. 